\title{
EFICIÊNCIA TÉCNICA AGROPECUÁRIA E DESMATAMENTO: ANÁLISE ESPACIAL PARA A AMAZÔNIA LEGAL BRASILEIRA ${ }^{\circ}$
}

\author{
AGRICULTURAL TECHNICAL EFFICIENCY AND \\ DEFORESTATION: SPATIAL ANALYSIS FOR THE BRAZILIAN \\ LEGAL AMAZON \\ Jéssica de Lima da Vida Pellenz* \\ Mariza de Almeida** \\ Viviani Silva Lirio ${ }^{* * *}$
}

aceptado: 16 octubre 2020 - aprobado: 07 enero 2021

\begin{abstract}
Resumo
A performance do setor agropecuário é essencial para o desempenho econômico brasileiro. O protagonismo do Brasil como exportador de commodities, contudo, exige atenção ao que se refere à contrapartida ambiental provocada, por se sustentar na expansão do uso da terra e não na eficiência produtiva. Percebendo a relevância da agropecuária para a economia brasileira e reconhecendo a importância de se promover um crescimento sustentável, este trabalho avalia como os níveis de eficiência agropecuária municipal se relacionam com o desmatamento na Amazônia Legal brasileira. Para tanto, foram utilizados métodos de Análise por Envoltória de Dados e de econometria espacial. Os resultados sugerem que os municípios com

- Pellenz, J. L. V., Almeida, M., \& Lirio, V. S. (2021). Eficiência técnica agropecuária e desmatamento: análise espacial para a Amazônia legal brasileira. Estudios económicos, 38(77), pp. 119-146. DOI: https://doi.org/10.52292/j.estudecon.2021.2200

* Universidade Federal de Viçosa, Brasil. ORCID: https://orcid.org/0000-0001-7091-0964. E-mail: jessipellenz@gmail.com

** Universidade de São Paulo, Brasil. ORCID: https://orcid.org/0000-0003-2910-8704. Brasil.

E-mail: mariza.almeida@usp.br

*** Universidade Federal de Viçosa, Brasil. ORCID: https://orcid.org/0000-0001-6806-819X.

E-mail: vslirio@ufv.br.
\end{abstract}


baixa eficiência contribuem para o aumento do desmatamento, enquanto que os mais eficientes contribuem para a redução nas áreas desmatadas.

Classificação JEL: Q15, R12, C14.

Palavras-chave: eficiência técnica, desmatamento, Amazônia Legal Brasileira, análise espacial.

\begin{abstract}
The agricultural sector's performance is essential for Brazilian economic performance. However, it requires attention to the inevitable environmental counterpart caused by the expansion of land use instead of productive efficiency. Realizing the relevance of agriculture to the Brazilian economy and recognizing the importance of promoting sustainable growth, this research assesses how the levels of counties' agricultural efficiency are related to deforestation in the Brazilian Legal Amazon. For that purpose, Data Envelopment Analysis and spatial econometrics were used. The results suggest that counties with a low level of efficiency contribute to the increase in deforestation, while the most efficient ones contribute to the reduction in deforested areas.
\end{abstract}

JEL Codes: Q15, R12, C14.

Keywords: technical efficiency, deforestation, Brazilian Legal Amazon. spatial analysis. 


\section{INTRODUÇÃO}

O Brasil figura entre os países que possuem as maiores reservas naturais do mundo. Esta posição, naturalmente, o torna o centro de muitos debates internacionais, vários deles limítrofes a questões relacionadas com a soberania nacional no trato com suas extensas áreas verdes e mananciais, entendidos esses, por muitos, como patrimônio supranacional, em virtude de suas interações com o meio ambiente mundial. Abrangendo extensa área do território brasileiro, a Amazônia Legal é exemplo de área de reserva natural composta por nove estados brasileiros. Engloba, além do bioma amazônico, parte do cerrado e do pantanal mato-grossense, áreas reconhecidamente sensíveis à intervenção humana, de grande biodiversidade animal e sede de muitas comunidades tradicionais (grupos que possuem sua organização social e que usam e ocupam territórios e recursos naturais para suas atividades).

A ocupação de novas áreas na Amazônia Legal com vistas à produção agropecuária tem sido alvo de intensos debates. Se, por um lado, essa expansão tem proporcionado um processo de elevação da produção agropecuária brasileira, por outro, é preciso reconhecer que esse aumento não decorre somente dos acréscimos de produtividade, mas, via de regra, por meio da degradação de extensas áreas florestais, criando legítimas preocupações em torno desse processo. Para Marchand (2012) e Pacheco (2009), os produtores, motivados pela potencial produtividade, tendem a expandir sua produção para terras com florestas e/ou sem uso produtivo.

No Brasil, o desbalanço entre contribuição produtiva e danos ambientais pode ser observado muito diretamente, já que o país está entre os maiores produtores e exportadores mundiais de produtos agrícolas, em particular as commodities (a exemplo da soja, do açúcar, das carnes de frangos e suínos, do café e da carne bovina) e, ao mesmo tempo, é uma das regiões com as maiores taxas de desmatamento, que é um dos fatores que mais impulsionam a emissão global de gases de efeito estufa.

Essa realidade pode ser confirmada por meio de indicadores bastante objetivos. O Brasil apresentou taxa de crescimento de $154 \%$ na produção de soja no período compreendido entre 2000 e 2018 e, no mesmo período, de $26 \%$ no rebanho bovino. Essa expansão consolidou o Brasil como segundo maior produtor de soja e de carne bovina do mundo (FAO, 2020; IBGE, 2020c). Em contrapartida, no mesmo intervalo temporal, a Amazônia Legal presenciou uma taxa de 59\% de crescimento em sua área desmatada (passou de $121181 \mathrm{~km}^{2}$ para $193095 \mathrm{~km}^{2}$ ). Essa elevação no desmatamento dos últimos dezoito anos apresenta muitas consequências, 
dentre as quais se destacam as alterações climáticas e na umidade do ar, redução na água doce proveniente de nascentes, erosão do solo e mudança no habitat dos animais (Marchand, 2012). Além disso, com a população cada vez mais preocupada com as questões ambientais, a demanda por produtos provenientes de áreas degradadas pode reduzir nos próximos anos, o que teria impacto direto sobre o agronegócio da região.

Nesse contexto de percepção de excludência entre produção e conservação ambiental, abre-se espaço para questionar os caminhos possíveis para conciliar o aumento da produção em áreas como a Amazônia Legal e as estratégias de proteção do meio ambiente, com base na adequação das técnicas utilizadas e na eficiência produtiva. Esta pesquisa insere-se exatamente neste espaço, qual seja o entendimento de que é necessário expandir o conhecimento acerca da possibilidade de se garantir o ritmo produtivo sem comprometer as áreas ambientais. Essa investigação está relacionada com a hipótese de que um aumento da eficiência produtiva (melhores tecnologias e combinação otimizada de insumos) reduz a necessidade de aumentar as áreas exploradas. Em outras palavras, produzir de forma eficiente evitaria uma expansão ainda maior da agropecuária sobre áreas de floresta na região da Amazônia Legal. Assim, tem-se por objetivo central analisar a eficiência técnica agropecuária dos municípios integrantes da Amazônia Legal Brasileira em 2017, verificando se há relação entre os escores de eficiência técnica e os níveis de desmatamento.

O artigo está estruturado em quatro seções, além desta introdução. A segunda seção aborda a expansão da fronteira agropecuária e seus impasses sobre o desmatamento da região da Amazônia Legal. Na terceira, são apresentadas as estratégias metodológicas e a base de dados utilizada no estudo. Na quarta seção, apresentam-se os resultados e as discussões e, por fim, são destacadas as principais conclusões acerca das implicações da pesquisa.

\section{A EXPANSÃO DA FRONTEIRA AGROPECUÁRIA E SEUS IMPASSES SOBRE O DESMATAMENTO}

As fronteiras agropecuárias brasileiras, naturalmente, sempre foram dinâmicas, movendo-se de acordo com um amplo conjunto de variáveis que vão desde a interiorização do território até as conquistas tecnológicas no trato com as diferentes áreas disponíveis para cultivo. Assim, com a expansão da produção agropecuária (bovinos, ovinos, bubalinos, soja, milho, café, cana-de-açúcar, arroz etc.), as fronteiras agropecuárias no Brasil sofreram transformações nas últimas décadas e muitas tentativas de reajuste foram providenciadas. 
Podem-se citar, como expansões relevantes, a mudança de extensas áreas da cafeicultura dos estados de São Paulo, Rio de Janeiro e Minas Gerais para o estado do Paraná, e a expansão da sojicultura dos estados de Rio Grande do Sul, Santa Catarina e Paraná para as regiões centro-oeste e norte (com destaque para Mato Grosso do Sul, Mato Grosso, Goiás e Rondônia). Além desses movimentos, a partir de 1987, um recorte geográfico específico foi observado no centro-norte do país, sendo considerado uma nova fronteira agropecuária, nomeada MATOPIBA, junção das siglas dos estados do Maranhão, Tocantins, Piaú e Bahia (Bacha, 2018).

Nesse mesmo contexto, emerge a criação formal da Amazônia Legal Brasileira, reconhecida a partir da promulgação da Lei $n^{\circ} 11.952$ de 2006, criada com o intuito de a União ${ }^{1}$ articular ações, em um mesmo complexo geoeconômico e social, que visem o desenvolvimento e redução das desigualdades regionais nos seus estados constitutivos (IBGE, 2020a). Em 2017, a Amazônia Legal apresentava 856852 estabelecimentos agropecuários, com 2962632 pessoas ocupadas e um valor de produção (animal e vegetal) de R $\$ 962$ 560. Do total desses estabelecimentos, apenas $17 \%$ já fizeram algum uso de adubação; $26 \%$ utilizaram agrotóxico; $5 \%$ usaram irrigação; $8 \%$ possuíam trator; e, $11 \%$ obtiveram financiamento, indicando baixo uso de estratégias de apoio ao aumento da eficiência técnica da produção agropecuária (IBGE, 2020b).

Além disso, a Amazônia Legal está localizada na maior floresta tropical do mundo, uma região que vem sofrendo com altas taxas de desmatamento derivadas, em grande parte, da busca por maiores níveis de produção. Almeida et al. (2016) ressaltam que o desflorestamento na região amazônica foi, em grande parte, convertido em pastagens, vegetação secundária e agricultura (culturas anuais). Observa-se, dessa forma, que existe um aparente trade-off entre produção agropecuária e preservação florestal; todavia, com o desenvolvimento de tecnologias e melhores práticas, acredita-se ser possível aumentar a produção sem necessitar expandir a área utilizada, tornando a produção mais eficiente e sustentável.

A Figura 1 expõe a evolução do efetivo do rebanho bovino, da área plantada de soja e da área desmatada na região Amazônia Legal. Nota-se que, no período de 2000 até 2018, a região Amazônia Legal apresentou crescimento no efetivo do rebanho de bovinos, na área plantada de soja e na área desmatada. O desmatamento passou de $121181 \mathrm{~km}^{2}$ para $193095 \mathrm{~km}^{2}$ (taxa de crescimento de 59\%); a área plantada de soja passou de $31579 \mathrm{~km}^{2}$ para $121047 \mathrm{~km}^{2}$ (taxa de crescimento de 283\%); e o efetivo do rebanho bovino passou de 47 mil cabeças para 86 mil (taxa de crescimento de 83\%).

1 Artigo 43 da Constituição Federal. 
Figura 1. Evolução do rebanho de gado bovino, área de soja e área desmatada na Amazônia Legal.

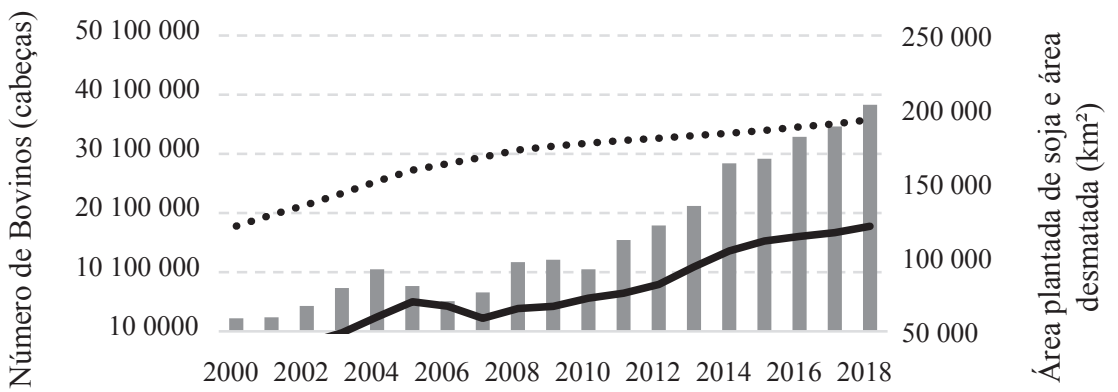

Efetivo do rebanho bovino _úrea plantada ...... Desmatamento

Fonte: elaborado pelos autores com base em IBGE (2020c).

Para Stabile et al. (2020), existem quatro estratégias que podem diminuir o desmatamento e aumentar a produção agropecuária: (a) eliminar a apropriação e especulação de terras através da designação de florestas públicas; (b) reduzir o desmatamento em propriedades privadas por meio do Código Florestal vigente no Brasil; (c) incentivar o aumento da produtividade em propriedades de médio e grande porte por meio de investimentos; e, (d) promover melhorias econômicas, ambientais e sociais por meio de assistência técnica aos pequenos agricultores.

A terceira estratégia apresentada pelos autores Stabile et al. (2020), que propõe incentivar o aumento da produtividade em propriedades de médio e grande porte por meio de investimentos, está estreitamente relacionada com a eficiência produtiva e converge com os propósitos de investigação dessa pesquisa. De fato, a eficiência pode ser técnica (produtiva), econômica e/ou, ambiental. Marchand (2012) menciona que, havendo eficiência técnica, melhor manejo dos insumos para produção, entre eles o uso da terra, os impactos sobre o desflorestamento serão menores. Assim, destaca que o desafio do século XXI é aumentar a eficiência do uso de recursos naturais necessários para a produção agropecuária sem comprometer o meio ambiente e possivelmente mitigando eventuais danos já existentes pela vigência de modelos menos eficientes.

Vários trabalhos empíricos têm sido propostos para analisar os determinantes do desmatamento. Oliveira et al. (2011) analisaram a relação entre crescimento 
da renda e desmatamento baseados na Curva de Kuznets Ambiental. Os resultados obtidos indicam que essa relação para os municípios da Amazônia Legal tem formato de $N$, isto é, quando o Produto Interno Bruto per capita (PIB per capita) é baixo, o desmatamento é decrescente; conforme o PIB per capita aumenta, o desmatamento cresce e, quando o PIB per capita chega em níveis mais elevados, o desmatamento volta a decrescer. Arraes, Mariano e Simonassi (2012), por sua vez, testaram os efeitos de fatores socioeconômicos e a eficácia da ação governamental sobre o desmatamento, concluindo que ações governamentais e fatores socioeconômicos (educação, renda e cumprimento das leis) são eficazes na redução do desmatamento. Marchand (2012) analisou o impacto da eficiência técnica sobre a propensão dos estabelecimentos agropecuários aumentarem a área agrícola, constatando que a eficiência técnica apresenta formato de U na conversão de área agrícola, ou seja, quando as fazendas atingem o nível de $61 \%$ de eficiência, elas começam a utilizar mais terra para fins agrícolas. Contudo, apesar dos esforços analíticos sobre o tema, ainda existem poucos estudos relacionando eficiência produtiva e desmatamento, especialmente que desenvolvam análises espaciais, o que abre espaço para a análise ora pretendida.

\section{MATERIAL E MÉTODOS}

Para entender a relação entre desmatamento e eficiência técnica, propõe-se uma abordagem em duas etapas. Na primeira, os escores de eficiência técnica agropecuária municipal dos municípios da Amazônia Legal são calculados por meio da Análise por Envoltória de Dados (DEA). Em seguida, realizam-se as análises espaciais, compreendendo a Análise Exploratória de Dados Espaciais (AEDE) e as estimações de modelos com dependência espacial.

\section{II.1. Cálculo da eficiência técnica}

A lógica da eficiência técnica se origina da fronteira de produção sob a hipótese de que nem todos os produtores operam com combinações ótimas de insumos (Leibenstein, 1966). Os escores individuais (no caso desta pesquisa, municipais) são calculados com base nos produtores que realizaram as combinações de insumos mais eficientes. Ou seja, inicialmente é calculada a fronteira eficiente e, posteriormente, os demais produtores têm seus escores ponderados com base nos eficientes.

Os métodos mais comumente utilizados na literatura para o cálculo da eficiência são os de Análise de Fronteira Estocástica (SFA, por sua sigla em inglês), que consiste na estimação paramétrica dos escores de eficiência, e a Análise por 
Envoltória de Dados (DEA, por sua sigla em inglês), que é um método não paramétrico de programação linear como forma de aplicação do conceito de eficiência técnica apresentados por Koopmans (1951) e Farrel (1957).

O modelo DEA é aplicado, simultaneamente, para construir a fronteira de produção e obter os escores de eficiência de cada produtor (município). O modelo descreve um conjunto de dados contendo $K$ insumos e $M$ produtos para cada um dos $N$ produtores. Os vetores-coluna $x_{i}$ e $y_{i}$ representam os dados de insumos e produtos, respectivamente. A matriz de insumos $X_{K x N}$ e de produtos $Y_{M x N}$ representam os dados para todos os produtores da amostra. A solução ótima para retornos variáveis de escala (VRS, por sua sigla em inglês) e orientação para insumos é realizada por meio do seguinte problema de programação linear (Coelli, Rahman \& Thirtle, 2002):

$$
\begin{gathered}
\min _{\theta, \lambda} \theta, \\
\text { s.a. } \quad \theta x_{i}-X \lambda \geq 0, \\
Y \lambda-y_{i} \geq 0, \\
N 1 \lambda=1 \\
\lambda \geq 0
\end{gathered}
$$

em que $\theta$ é um escalar que representa o escore do $i$-ésimo produtor e satisfará $\theta \leq 1$, sendo igual a um quando pertencer à fronteira e, consequentemente, é um produtor tecnicamente eficiente. $\lambda$ é um vetor $N x 1$ de constantes, $N 1 \lambda$ é um vetor $N x 1$ de uns. O problema representado pela Equação (1) é resolvido $N$ vezes, de forma, que para cada produtor, seja auferido um valor correspondente de $\theta$.

A variável utilizada como produto na estimação dos escores de eficiência foi o Valor Bruto da Produção Agropecuária (VBP) municipal, que foi construída com base no Censo Agropecuário (IBGE) e compreende o somatório do VBP de lavouras permanentes e temporárias, bem como o VBP de pecuária bovina. Os insumos utilizados foram: a área total dos estabelecimentos agropecuários, a força de trabalho (representada pelo número total de trabalhadores empregados nas atividades agropecuárias), máquinas (tratores, máquinas e implementos agropecuários), gastos agropecuários (sementes, adubos e corretivos, compra de animais, agrotóxicos, medicamentos para animais, combustíveis, ração e suplementos).

\section{II.2. Relação eficiência versus desmatamento}

Após a estimação da eficiência técnica dos municípios, a etapa seguinte correspondeu à determinação do efeito dos escores de eficiência agropecuária sobre 
o desmatamento. Para tal propósito, foram utilizadas ferramentas de análise de dados espaciais e econometria espacial. A seguir, são apresentadas as metodologias aplicadas.

\section{II.2.1. Análise de autocorrelação espacial}

A fim de detectar a existência de dependência espacial nas variáveis, foi conduzida uma Análise Exploratória de Dados Espaciais (AEDE), que permitiu verificar se variáveis de uma região $i$ sofrem influência (ou influenciam) elementos de regiões vizinhas. Foram utilizadas as estatísticas $I$ de Moran Local e Global para análises univariadas (que relacionam uma variável $x$ do município $i$ à mesma variável do município vizinho $j$ ), e bivariadas (que determinam a dependência espacial entre variáveis distintas em diferentes municípios) (Anselin, 1995). O I de Moran Global é definido da seguinte forma:

$$
I=\frac{n}{S_{o}} \frac{\sum_{i} \sum_{j} w_{i j} z_{i} z_{j}}{\sum_{i=1}^{n} z_{i}^{2}}
$$

em que $I$ é o índice de Moran, $n$ representa o número de municípios analisados; $z$ são os valores padronizados da variável de interesse; $W z$ são os valores médios padronizados da variável de interesse nas localidades vizinhas, definidos de acordo com a matriz de ponderação $W$; $w_{i j}$ define a forma como o município $i$ está espacialmente correlacionado com a localidade $j ; S_{o}$ é um escalar igual à soma de todos os elementos de $\mathrm{W}$.

$\mathrm{O}$ valor esperado do $I$ de Moran é de $I^{*}=-[1 /(n-1)]$. Se o valor encontrado através da Equação (2) for igual ao valor esperado, rejeita-se a hipótese de autocorrelação espacial (Cliff; Ord, 1981). Valores de $I$ que excederem $I^{*}$ revelam uma autocorrelação espacial positiva, enquanto que valores abaixo de $I^{*}$ indicam autocorrelação espacial negativa (Almeida, 2012). A autocorrelação espacial local, indicada pelo $I$ de Moran local é dado por:

$$
I_{i}=\sum_{i} z_{i} \sum_{j} w_{i j} z_{j}=\sum_{i} \sum_{j} w_{i j} z_{i} z_{j}
$$

Matematicamente, a diferença entre as análises Global e Local é que a Equação (3) captura padrões locais de autocorrelação espacial, como pode ser observado pelo indicador $i$ do índice. Esse indicador é chamado de LISA (Local Indicator of 
Spatial Association) e avalia a associação espacial local e sua significância para cada município da análise.

\section{II.2.2. Estratégia econométrica}

Para que sejam avaliados os efeitos da eficiência técnica sobre o nível de desmatamento na Amazônia Legal Brasileira, foram realizadas estimações de modelos com a incorporação da dependência espacial. Inicialmente, estimou-se um modelo de regressão padrão por meio de Mínimos Quadrados Ordinários (MQO) e, posteriormente, realizou-se a incorporação de fatores de dependência espacial por meio dos modelos de Defasagem Espacial (SAR, por sua sigla em inglês) e de Erro Autorregressivo Espacial (SEM, por sua sigla em inglês). As especificações dos modelos se encontram na Tabela 1 .

Tabela 1. Resumo dos métodos de estimação utilizados

\begin{tabular}{lcc}
\hline \multicolumn{2}{c}{ Equação } & Modelo \\
\hline$y=X \beta+\varepsilon$, & $\varepsilon \sim N\left(0, \sigma^{2} I_{n}\right)$ & Mínimos Quadrados Ordinários (MQO) \\
$y=\rho W y+X \beta+\varepsilon$, & $\varepsilon \sim N\left(0, \sigma^{2} I_{n}\right)$ & Modelo de Lag Espacial (SAR) \\
$y=X \beta+\xi$, & $\xi=\lambda W \xi+\varepsilon$ & Modelo de Erro Espacial (SEM) \\
\hline
\end{tabular}

Fonte: elaborado pelos autores.

em que $y$ é um vetor $n \times 1$ de valores relativos à variável dependente (desmatamento), $\mathrm{X}$ é uma matriz $n \times k$ de variáveis explicativas (eficiência técnica e demais variáveis independentes) mais a constante, é um vetor $k x 1$ de coeficientes da regressão, e é um vetor $n \times 1$ relativo ao termo de erro aleatório. $W$ representa a matriz de contiguidade que descreve o padrão de vizinhança entre os municípios, $\rho$ é o coeficiente autorregressivo espacial do modelo SAR, e $\lambda$ é o coeficiente do erro espacial autorregressivo do modelo SEM.

Ambos os modelos (SAR e SEM) incorporam fatores de dependência espacial ao modelo padrão MQO e são considerados modelos globais. No entanto, se diferenciam quanto à forma de interação espacial. A especificação de SAR demonstra que a variável que apresenta dependência espacial é a própria variável depen- 
dente. No caso deste estudo, significa que o desmatamento de um município $i$ está relacionado com o desmatamento de municípios vizinhos. Já no modelo SEM, a relação de dependência espacial é residual, ou seja, há padrões espaciais manifestados nos termos de erro. Em outras palavras, existem fatores não modelados que são espacialmente autocorrelacionados. Dessa forma, o resíduo da regressão SEM não são homocedásticos e independentes espacialmente.

Para a seleção do modelo mais adequado para a análise, utilizou-se o processo de decisão sugerido por Anselin (2005), no qual são considerados os testes estatísticos LM-Error e LM-Lag. Caso nenhum dos testes rejeitar a hipótese nula, considera-se que não há dependência espacial e o modelo MQO é o mais indicado. Se apenas LM-Error rejeitar a hipótese nula, será escolhido o modelo SEM. Caso apenas LM-Lag rejeitar $H_{0}$, o modelo escolhido será o SAR. Contudo, se os dois testes rejeitarem $\mathrm{H}_{0}$, serão consideradas as estatísticas robustas dos testes.

Optou-se pela não inclusão de efeitos fixos espaciais pelo fato de que, uma vez que estes são capturados por uma variável constante, presume-se que o efeito influencie todas as observações dentro do seu grupo de maneira idêntica. Contudo, caso haja heterogeneidade ou interação dentro do grupo, esse fator será relegado ao termo de erro, resultando em distúrbios heterocedásticos. Anselin e Arribas-Bel (2013) argumentam ainda que, em geral, o uso de efeitos fixos espaciais não corrige adequadamente a presença de autocorrelação espacial à medida que viola um pressuposto importante da literatura da interação espacial, chamado de "Primeira Lei de Tobler", segundo a qual "everything depends on everything else, but closer things more so" (Tobler 1979).

\section{II.3. Delimitação territorial e fontes de dados}

Esta análise foi conduzida com dados do Censo Agropecuário 2017 e do Programa de Cálculo do Desflorestamento da Amazônia (PRODES) para municípios integrantes da Amazônia Legal brasileira. A escolha da delimitação territorial se justifica pelo fato de que, no Brasil, o maior causador de emissões de gases de efeito estufa está relacionado ao desmatamento na Amazônia. Segundo o MMA (2016), 78\% das emissões brasileiras se originaram do processo de mudança no uso da terra e florestas, sendo que, desse total, $65.2 \%$ foi proveniente da região da Amazônia Legal. A região geográfica a que se refere este estudo corresponde a uma superfície de aproximadamente $5217423 \mathrm{~km}^{2}$ e representa cerca de $61 \%$ do 
território brasileiro, sendo composta por 772 municípios pertencentes a 9 estados $^{2}$ brasileiros (IBGE, 2019). O Quadro 1 apresenta as variáveis utilizadas nas análises e suas respectivas unidades de medida e fontes.

Quadro 1. Variáveis e fonte de dados utilizadas nas estimações

\begin{tabular}{|c|c|c|}
\hline Variável & Descrição & Fonte \\
\hline $\begin{array}{l}\text { VBP } \\
\text { Agropecuário }\end{array}$ & $\begin{array}{l}\text { Valor Bruto da Produção das } \\
\text { lavouras permanentes e temporárias } \\
\text { e valor bruto da pecuária bovina. }\end{array}$ & $\begin{array}{c}\text { Censo } \\
\text { Agropecuário - IBGE }\end{array}$ \\
\hline $\begin{array}{l}\text { Área } \\
\text { Agropecuária }\end{array}$ & $\begin{array}{l}\text { Área dos estabelecimentos } \\
\text { agropecuários em hectares. }\end{array}$ & $\begin{array}{c}\text { Censo } \\
\text { Agropecuário - IBGE }\end{array}$ \\
\hline Gado Bovino & $\begin{array}{l}\text { Número de cabeças no rebanho } \\
\text { bovino. }\end{array}$ & $\begin{array}{c}\text { Censo } \\
\text { Agropecuário - IBGE }\end{array}$ \\
\hline $\begin{array}{l}\text { Força de } \\
\text { Trabalho }\end{array}$ & Número de trabalhadores ocupados. & $\begin{array}{c}\text { Censo } \\
\text { Agropecuário - IBGE }\end{array}$ \\
\hline $\begin{array}{l}\text { Força } \\
\text { Mecânica }\end{array}$ & $\begin{array}{l}\text { Número de tratores, implementos } \\
\text { e máquinas existentes nos } \\
\text { estabelecimentos agropecuários. }\end{array}$ & $\begin{array}{c}\text { Censo } \\
\text { Agropecuário - IBGE }\end{array}$ \\
\hline $\begin{array}{l}\text { Área } \\
\text { Desmatada }\end{array}$ & Área desmatada em $\mathrm{Km}^{2}$. & PRODES \\
\hline $\begin{array}{l}\text { Área com } \\
\text { Hidrografia }\end{array}$ & $\mathrm{Km}^{2}$ & PRODES \\
\hline $\begin{array}{l}\text { Corretivos e } \\
\text { Fertilizantes }\end{array}$ & $\begin{array}{l}\text { Número de estabelecimentos } \\
\text { agropecuários que utilizaram } \\
\text { corretivos e fertilizantes no solo. }\end{array}$ & $\begin{array}{c}\text { Censo A } \\
\text { gropecuário - IBGE }\end{array}$ \\
\hline
\end{tabular}

2 Estados integrantes da Amazônia Legal brasileira: Acre, Amapá, Amazonas, Maranhão, Mato Grosso, Pará, Rondônia, Roraima e Tocantins. 


\begin{tabular}{|c|c|c|}
\hline Pesticidas & $\begin{array}{c}\text { Número de estabelecimentos } \\
\text { agropecuários que utilizaram } \\
\text { pesticidas. }\end{array}$ & $\begin{array}{c}\text { Censo } \\
\text { Agropecuário - IBGE }\end{array}$ \\
\hline Eletricidade & $\begin{array}{l}\text { Número de estabelecimentos } \\
\text { agropecuários com uso de } \\
\text { eletricidade. }\end{array}$ & $\begin{array}{c}\text { Censo } \\
\text { Agropecuário - IBGE }\end{array}$ \\
\hline $\begin{array}{l}\text { Conservação } \\
\text { do Solo }\end{array}$ & $\begin{array}{l}\text { Número de estabelecimentos } \\
\text { agropecuários que realizaram } \\
\text { preparo/conservação do solo. }\end{array}$ & $\begin{array}{c}\text { Censo } \\
\text { Agropecuário - IBGE }\end{array}$ \\
\hline Financiamento & $\begin{array}{l}\text { Número de estabelecimentos } \\
\text { agropecuários que se utilizaram de } \\
\text { financiamento agrícola. }\end{array}$ & Censo Agropecuário - IBGE \\
\hline
\end{tabular}

Fonte: elaborado pelos autores.

\section{RESULTADOS}

\section{III.1. Estimação dos Escores de Eficiência}

Para a atender ao objetivo de analisar os efeitos da eficiência agropecuária dos municípios sobre o desmatamento da Amazônia Legal em 2017, realizou-se, inicialmente, a estimação dos escores de eficiência técnica através da Análise por Envoltória de Dados (DEA, por sua sigla em inglês). Os resultados obtidos revelaram uma grande discrepância entre os escores de eficiência dos municípios estudados, que apresentam uma distribuição de frequência entre 0.01 e 1.00. Os dados da Tabela 2 descrevem as estatísticas descritivas dos escores de eficiência técnica.

Como indicado, a eficiência média na região estudada é de 0.24 , mostrando que a atividade agropecuária na Amazônia Legal é ineficiente. Os escores de eficiência variam bastante entre municípios do mesmo estado e entre estados, conforme mostram os resultados dos desvios-padrão estaduais e total. Os estados com maior grau de ineficiência são Rondônia, Roraima, Maranhão e Acre, que possuem escores médios abaixo de 0.2. Já os estados com melhor desempenho são Amazonas e Pará, sendo os únicos a alcançar escores maiores do que a média da região. A Figura 2 apresenta a distribuição espacial dos escores de eficiência técnica, bem como a distribuição de frequência dos resultados. 
Tabela 2. Resultados da estimação da eficiência técnica por estado

\begin{tabular}{llllll}
\hline Estado & $\begin{array}{l}\text { Número de } \\
\text { Eficientes }\end{array}$ & Média & Mín & Máx & D.P.* \\
\hline Acre & 0 & 0.19 & 0.03 & 0.58 & 0.19 \\
\hline Amazonas & 7 & 0.37 & 0.03 & 1.00 & 0.32 \\
\hline Amapá & 0 & 0.22 & 0.07 & 0,62 & 0.15 \\
\hline Maranhão & 2 & 0.18 & 0.04 & 1,00 & 0.16 \\
\hline Mato & 7 & 0.35 & 0.02 & 1,00 & 0.29 \\
Grosso & 7 & 0.25 & 0.01 & 1,00 & 0.23 \\
Pará & 7 & 0.09 & 0.02 & 0,31 & 0.07 \\
\hline Rondônia & 0 & 0.13 & 0.05 & 0,34 & 0.08 \\
\hline Roraima & 0 & 0.20 & 0.03 & 0,88 & 0.15 \\
\hline Tocantins & 0 & 0.24 & 0.01 & 1.00 & 0.22 \\
\hline Total & 23 & & & & \\
\hline Notal & & 0.03 & \\
\hline
\end{tabular}

Nota: *Desvio Padrão

Fonte: resultados da pesquisa.

Apenas 3\% dos municípios da amostra (23 dentre os 733) foram considerados eficientes (escore igual a 1) e distribuem-se em 4 dos 9 estados ( 7 no Amazonas, 7 no Mato Grosso, 7 no Pará e 2 no Maranhão). A maior parte dos municípios (434) apresenta escores de eficiência muito pequenos (área em branco). Percebe-se que há uma área grande do território (que corresponde a todo o território de Roraima e boa parte dos estados de Amazonas e Pará) em que há predominância de municípios com escores de eficiência muito baixos (menores que 0.2 ) e que se situam muito próximos uns dos outros. Diversos fatores justificam esses resultados, dentre eles, a presença de Unidades de Conservação e tipos de solo impróprios para atividades agropecuárias.

Em complemento a essas análises iniciais, são apresentados, a seguir, os resultados e discussões acerca da análise exploratória de dados espaciais compreendendo as variáveis desmatamento e eficiência técnica agropecuária. 
Figura 2. Escores de eficiência técnica: distribuição espacial de frequência

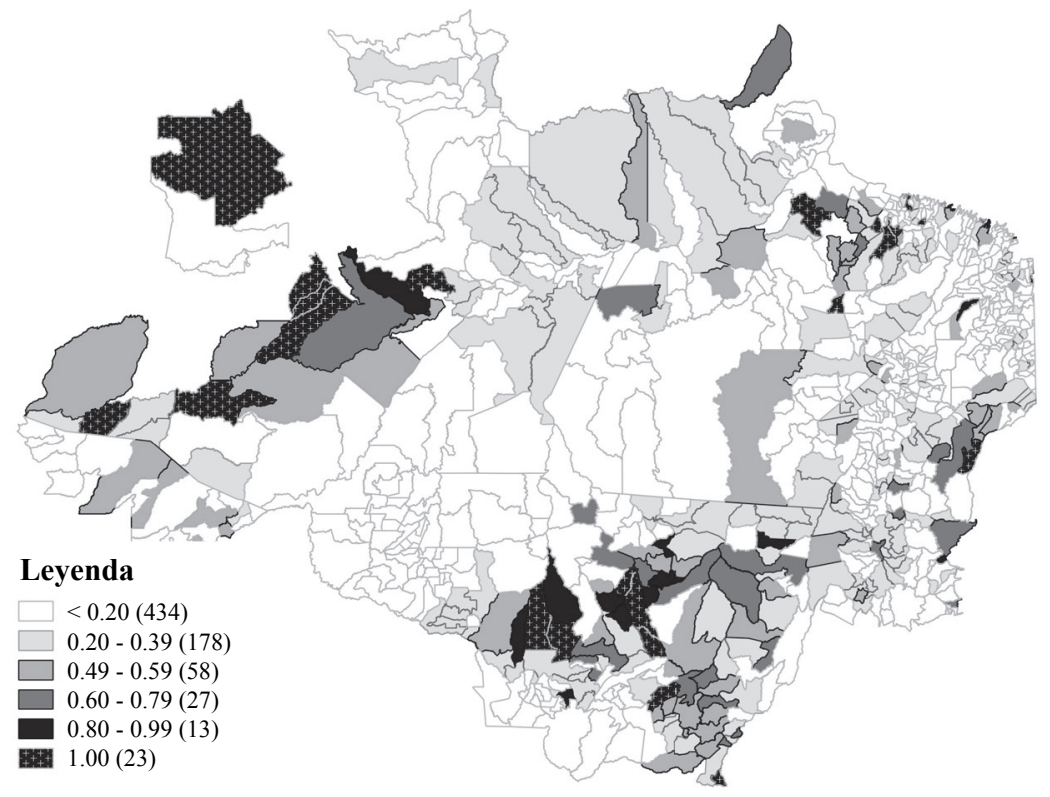

Fonte: resultados da pesquisa.

III.2. Análise exploratória de dados espaciais (AEDE)

Essa análise tem início com a verificação da presença de autocorrelação espacial realizada através do Índice de Moran. Foram estimados os índices com três tipos de convenção (Rainha de grau 1, Rainha de grau 2 e Torre). Os resultados estão apresentados na Tabela 3.

Os resultados apresentados na Tabela 3 demonstram que, para todas as convenções testadas, os índices reportados são maiores do que o valor crítico $\left(I^{*}=0.00137\right)$, revelando a presença de autocorrelação espacial para as duas variáveis em análise (eficiência técnica e desmatamento). Além disso, com $p$-valores próximos de zero, pode-se concluir que os resultados são estatisticamente significativos. A Figura 3 reporta os resultados de autocorrelação espacial local univariada (LISA) para a variável de eficiência técnica. 
Tabela 3. Índices de Moran

\begin{tabular}{|c|c|c|c|}
\hline & Rainha 1 & Rainha 2 & Torre \\
\hline $\begin{array}{l}\text { Moran Univariado } \\
\text { (Desmatamento) }\end{array}$ & $\begin{array}{l}0.61 \\
p \text {-Valor: } 0.001\end{array}$ & $\begin{array}{l}0.47 \\
p \text {-Valor: } 0.001\end{array}$ & $\begin{array}{l}0.61 \\
p \text {-Valor: } 0.001\end{array}$ \\
\hline $\begin{array}{l}\text { Moran Univariado } \\
\text { (Eficiência) }\end{array}$ & $\begin{array}{l}0.32 \\
p \text {-Valor: } 0.001\end{array}$ & $\begin{array}{l}0.22 \\
p \text {-Valor: } 0.001\end{array}$ & $\begin{array}{l}0.14 \\
p \text {-Valor: } 0.001\end{array}$ \\
\hline $\begin{array}{l}\text { Moran Bivariado } \\
\text { (Desmatamento x } \\
\text { Eficiência) }\end{array}$ & $\begin{array}{l}-0.18 \\
p \text {-Valor: } 0.001\end{array}$ & $\begin{array}{l}-0.17 \\
p \text {-Valor: } 0.001\end{array}$ & $\begin{array}{l}-0.18 \\
p \text {-Valor: } 0.001\end{array}$ \\
\hline
\end{tabular}

Fonte: resultados da pesquisa.

Os resultados apresentados na Figura 3(b) mostram que 176 municípios possuem agrupamentos (clusters) espaciais estatisticamente significativos para a eficiência técnica. Na Figura 3(a), verifica-se que o número de municípios em agrupamentos alto-alto e baixo-baixo é de 151. Esses resultados indicam que, nessa faixa geográfica (áreas em cor preta sólida e pontilhada), municípios com altos escores de eficiência situam-se próximos a municípios com a mesma característica, e municípios com baixos escores são vizinhos de outros municípios tão ineficientes quanto. Já os agrupamentos alto-baixo e baixo-alto contabilizam 25 municípios e revelam que municípios com altos escores de eficiência se avizinham de municípios com baixos escores, e vice-versa.

Essa primeira etapa de análise revela um baixo desempenho da eficiência técnica agropecuária na região da Amazônia Legal brasileira. Esses resultados não apenas corroboram os encontrados por Silva, Perrin e Fulginiti (2016) ${ }^{3}$, como indica uma manutenção (e, possivelmente, uma redução) dos parcos resultados de escores de eficiência agropecuária de 2006 para 2017. Além disso, verificaram-se alguns padrões espaciais importantes, especialmente no que diz respeito ao cluster baixo-baixo (Figuras 2 e 3), que inclui boa parte dos municípios dos estados do Maranhão, Rondônia e Mato Grosso. É importante ressaltar que o estado do Mato Grosso é um dos principais atores do agronegócio brasileiro, sendo necessária atenção não apenas para seus níveis de produtividade, mas também para os possíveis impactos que a produção agropecuária possa causar ao meio ambiente. A Figura 4 apresenta a análise de autocorrelação espacial (LISA) para desmatamento.

3 Silva, Perrin e Fulginiti (2016) analisam a eficiência técnica para a ALB no ano de 2006, contudo utilizam SFA para o cálculo dos escores. 
Figura 3. Mapas LISA e significância (escores de eficiência técnica)

a)

Leyenda

Alto-Alto

E:9 Baixo-Baixo

Baixo-Alto

:::- Alto-Baixo

Não Significativos

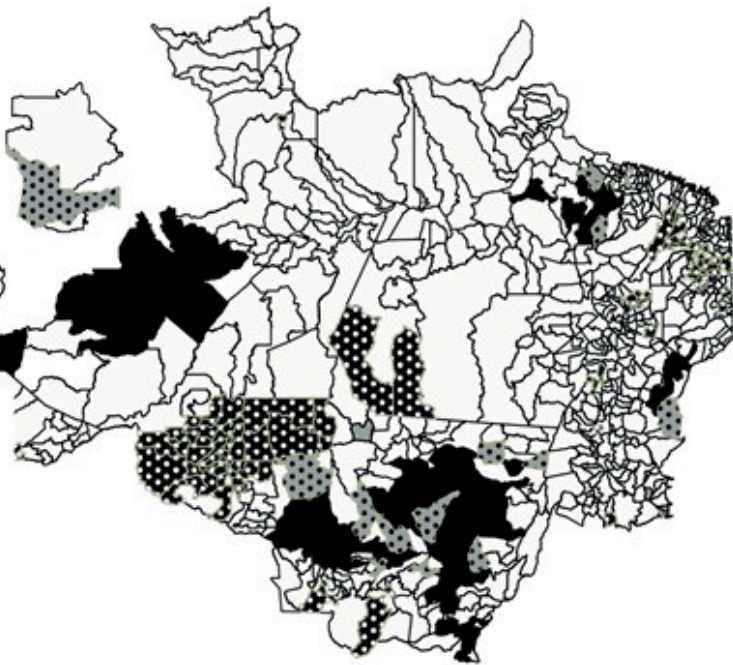

b)

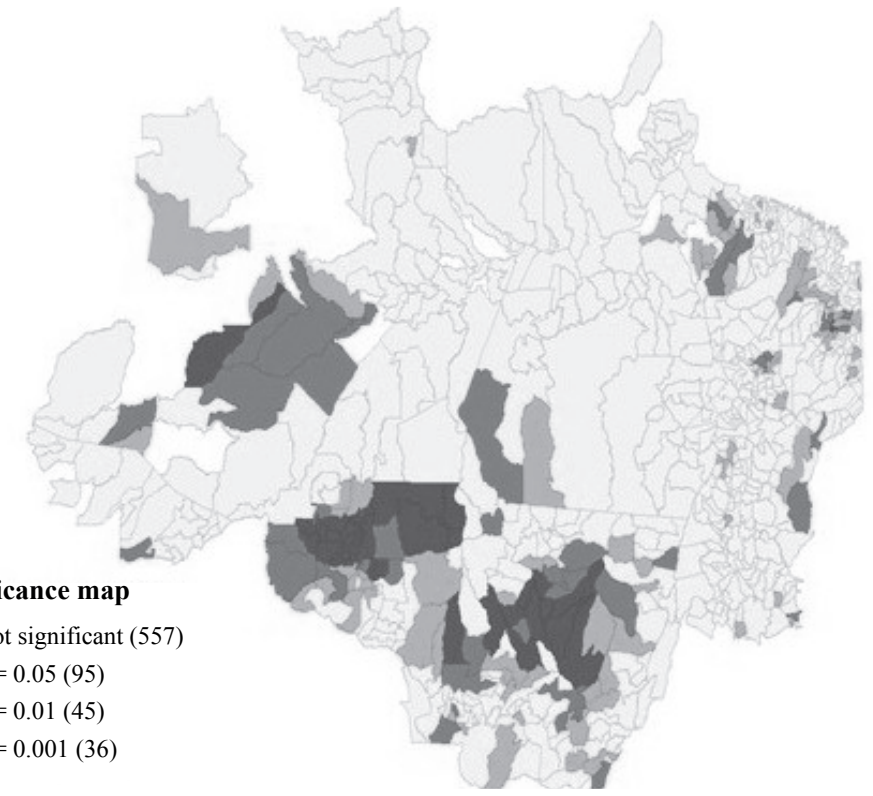

Fonte: resultados da pesquisa. 
Figura 4. Mapas LISA e significância (escores desmatamento)
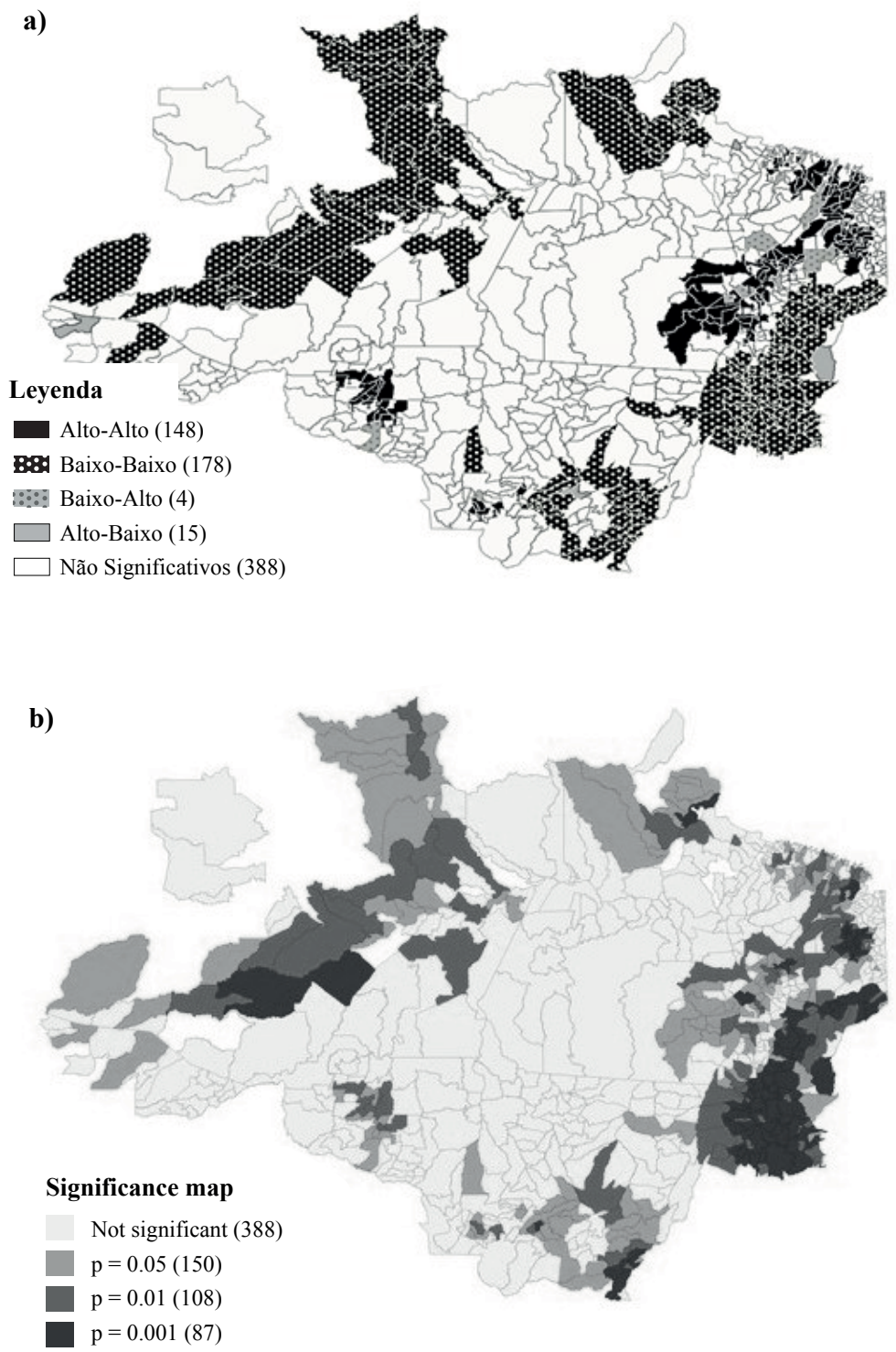

Fonte: resultados da pesquisa. 
Figura 5. Mapas BiLISA (escores de eficiência versus desmatamento)

a)

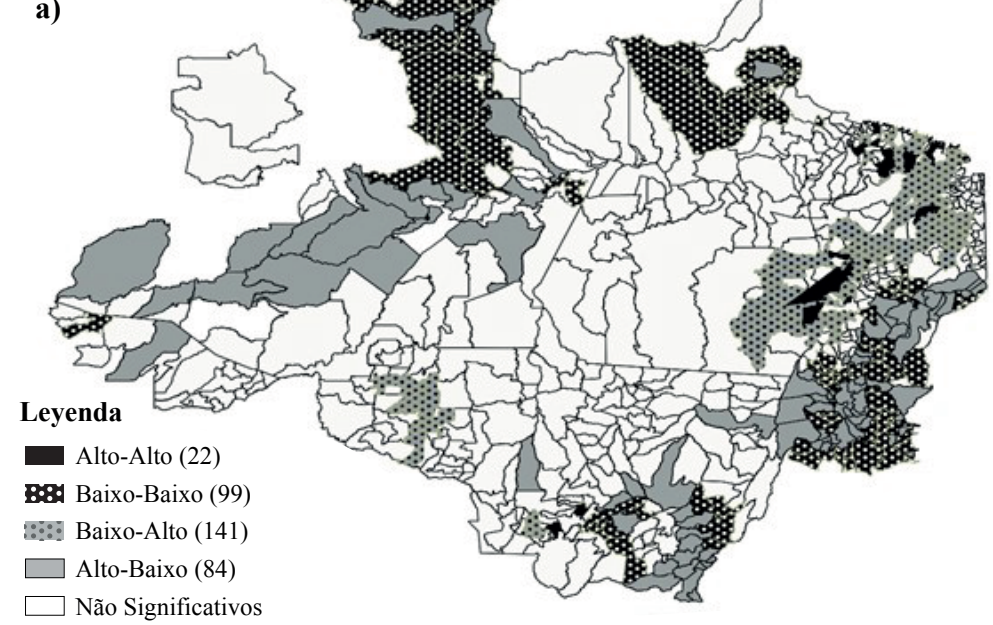

b)

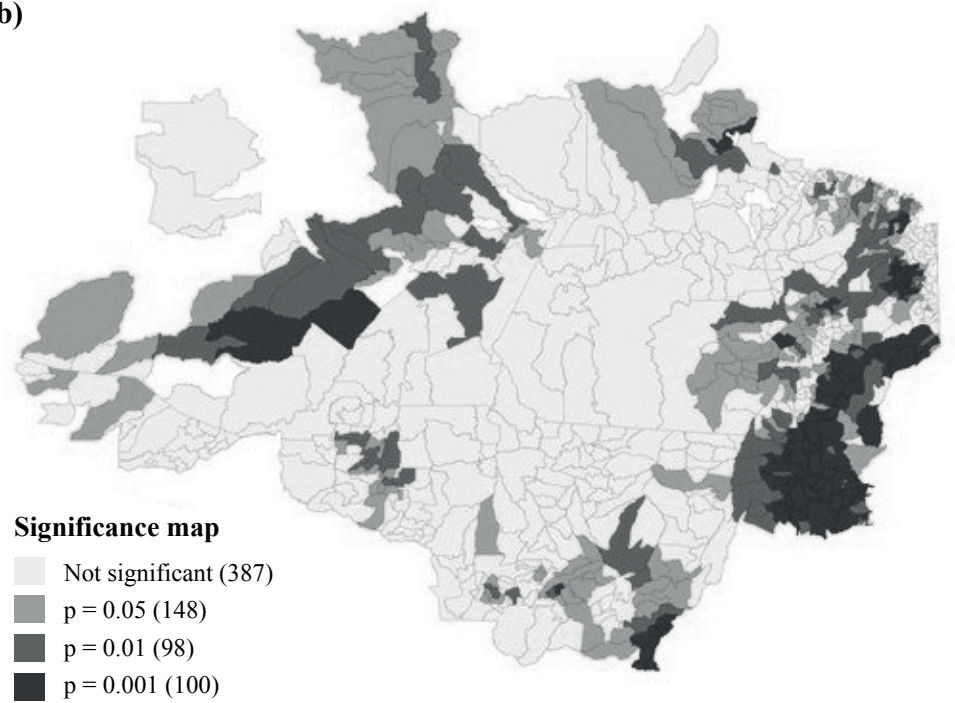

Fonte: resultados da pesquisa. 
A análise de autocorrelação espacial para desmatamento destaca 345 municípios com agrupamentos (clusters) espaciais estatisticamente significativos (Figura 4-b). Os agrupamentos alto-baixo e baixo-alto são compostos por 19 municípios, o que caracteriza dissimilaridade entre si, ou seja, municípios com alto nível de desmatamento se localizam próximos a outros em que há baixo desmatamento, e vice-versa. A distribuição espacial do desmatamento revela uma característica de agrupamento, em especial, que precisa de uma avaliação cuidadosa e de atenção por parte das autoridades. Trata-se das regiões apontadas como cluster alto-alto e que se concentram, quase que totalmente, nos estados do Maranhão, Pará, Tocantins e Rondônia, nos quais os escores de eficiência técnica são menores. Esse cluster é caracterizado por 148 municípios e mostra que os municípios com maior nível de desmatamento estão situados próximos uns dos outros. Já o cluster Baixo-Baixo agrega 178 municípios e se caracteriza pelo baixo nível de desmatamento, ocupando regiões afastadas do centro da área estudada (região em preto pontilhado).

Para melhor especificar a correlação espacial entre desmatamento e eficiência, e verificar uma primeira relação entre essas variáveis, é possível analisar os resultados apresentados na Figura 5, que indicam que 141 municípios possuem característica de baixa eficiência e altos níveis de desmatamento, o que corresponde a quase $20 \%$ do território da Amazônia Legal Brasileira. Para além disso, um cluster alto-baixo também significativo e representativo ( $11 \%$ do território analisado) apresenta mais uma evidência de que há uma correlação negativa entre as variáveis em questão.

Para comparar os escores de eficiência técnica estimados com o nível de desmatamento, foi realizada a análise de autocorrelação espacial local bivariada (BiLISA, por sua sigla em inglês). Os resultados estão expressos na Figura 5.

A Figura 5(b) mostra que 346 municípios pertencem a agrupamentos espaciais estatisticamente significativos. Percebe-se um cluster bivariado alto-alto de 22 municípios, localizados principalmente no estado do Pará, e composto por municípios com altos escores de eficiência e elevados níveis de desmatamento. Outro cluster bivariado, do tipo baixo-baixo, compreende municípios com escores de eficiência pequenos e que estão próximos de municípios com baixo nível de desmatamento. $\mathrm{O}$ cluster bivariado baixo-alto compreende municípios, principalmente, dos estados do Pará, Tocantins, Maranhão e Rondônia. Nesse agrupamento, os municípios com elevados escores de eficiência técnica fazem vizinhança com municípios com baixos níveis de desmatamento. A composição desse cluster coincide com o agrupamento local univariado do desmatamento (Figura 4a), o qual dispõe os municípios com baixos níveis de desmatamento próximos uns dos outros. Existe, 
ainda, um cluster bivariado do tipo alto-baixo: municípios com baixos escores de eficiência técnica próximos de municípios com níveis altos de desmatamento.

III.3. Estimação dos modelos espaciais

A análise exploratória de dados espaciais realizada revelou a existência de autocorrelação espacial em nível municipal para as variáveis desmatamento e eficiência técnica, bem como para a combinação de ambas. A partir desse resultado inicial, deu-se início à análise para verificar se os escores de eficiência técnica agropecuária na Amazônia Legal impactam nos níveis de desmatamento dessa região. A fim de trazer mais luz ao que diz respeito à relação entre as duas variáveis, analisam-se os resultados das estimações apresentadas na Tabela 4.

Inicialmente, foi estimado o modelo por Mínimos Quadrados Ordinários para o desmatamento, como etapa preliminar para as estimações dos modelos econométricos espaciais. Na Tabela 4, são apresentados os resultados das estimações, bem como medidas de qualidade de ajuste para a comparação entre os modelos. Tais medidas se referem ao log likelihood e aos critérios de Akaike (AIC) e Schwarz (BIC).

Tabela 4 - Resultados das estimações dos modelos espaciais

Variável dependente: Área Desmatada (\%)

\begin{tabular}{|c|c|c|c|}
\hline Variáveis & MQO & SAR & SEM \\
\hline Constante & $\begin{array}{c}1.28254 * * * \\
(0.1968)\end{array}$ & $\begin{array}{c}1.1277 * * * \\
(0.1761)\end{array}$ & $\begin{array}{c}1.1870 * * * \\
(0.1733)\end{array}$ \\
\hline Escore & $\begin{array}{c}0.5763 * * \\
(0.2774)\end{array}$ & $\begin{array}{c}0.5941^{* *} \\
(0.2461)\end{array}$ & $\begin{array}{c}0.6364 * * * \\
(0.2319)\end{array}$ \\
\hline Escore $^{2}$ & $\begin{array}{c}-0.7149 * * \\
(0.3118)\end{array}$ & $\begin{array}{c}-0.7494 * * * \\
(0.2766)\end{array}$ & $\begin{array}{c}-0.8196 * * * \\
(0.2581)\end{array}$ \\
\hline PIB agro pc (ln) & $\begin{array}{c}-0.0710^{* *} \\
(0.0318)\end{array}$ & $\begin{array}{l}-0.0523 * \\
(0.0282)\end{array}$ & $\begin{array}{l}-0.0340 \\
(0.0271)\end{array}$ \\
\hline Área Agropec (ln) & $\begin{array}{c}-0.1782 * * * \\
(0.0229)\end{array}$ & $\begin{array}{c}-0.1469 * * * \\
(0.0208)\end{array}$ & $\begin{array}{c}-0.1618 * * * \\
(0.0213)\end{array}$ \\
\hline Gado (ln) & $\begin{array}{c}0.1018 * * * \\
(0.0192)\end{array}$ & $\begin{array}{c}0.0762 * * * \\
(0.0174)\end{array}$ & $\begin{array}{c}0.0925 * * * \\
(0.0181)\end{array}$ \\
\hline
\end{tabular}




\begin{tabular}{|c|c|c|c|}
\hline Corretivos e Fertilizantes & $\begin{array}{c}0.0328 * * \\
(0.0134)\end{array}$ & $\begin{array}{c}0.0345 * * * \\
(0.0118)\end{array}$ & $\begin{array}{c}0.0255^{* *} \\
(0.0118)\end{array}$ \\
\hline Pesticidas & $\begin{array}{c}-0.0344 * * \\
(0.0143)\end{array}$ & $\begin{array}{c}-0.0303 * * \\
(0.0127)\end{array}$ & $\begin{array}{c}-0.0365 * * * \\
(0.0123)\end{array}$ \\
\hline Eletricidade & $\begin{array}{l}0.0464 * \\
(0.0256)\end{array}$ & $\begin{array}{c}0.0313 \\
(0.0228)\end{array}$ & $\begin{array}{c}0.0491 * * \\
(0.0223)\end{array}$ \\
\hline Conservação do solo & $\begin{array}{c}0.0271 \\
(0.0167)\end{array}$ & $\begin{array}{c}0.0239 \\
(0.0148)\end{array}$ & $\begin{array}{c}0.0188 \\
(0.0139)\end{array}$ \\
\hline Financiamento & $\begin{array}{c}0.0242 \\
(0.0203)\end{array}$ & $\begin{array}{c}0.0175 \\
(0.0180)\end{array}$ & $\begin{array}{l}0.0302 * \\
(0.0180)\end{array}$ \\
\hline Hidrografia & $\begin{array}{c}-0.0452 * * * \\
(0.0058)\end{array}$ & $\begin{array}{c}-0.0386 * * * \\
(0.0053)\end{array}$ & $\begin{array}{c}-0.0455 * * * \\
(0.0055)\end{array}$ \\
\hline$\rho$ & & $\begin{array}{c}0.3312 * * * \\
(0.0439)\end{array}$ & \\
\hline$\lambda$ & & & $\begin{array}{c}0.4731 * * * \\
(0.0504)\end{array}$ \\
\hline $\mathrm{R}^{2}$ & 0.4688 & 0.5658 & 0.6043 \\
\hline Log likelihood & 18.95 & 44.18 & 51.47 \\
\hline Critério Akaike (AIC) & -13.89 & -62.37 & -78.94 \\
\hline Critério Schwarz (BIC) & 30.95 & -13.79 & -34.11 \\
\hline Diagnósticos de $M Q O$ & $D F$ & Valor & P-Valor \\
\hline Normalidade dos erros (Jarque-Bera) & 2 & 2.7566 & 0.2520 \\
\hline Heteroscedasticidade (Breusch-Pagan) & 11 & 31.4620 & 0.00093 \\
\hline $\begin{array}{l}\text { Número de Condição de } \\
\text { Multicolinearidade }\end{array}$ & 21.0694 & & \\
\hline Multiplicador de Lagrange (Lag) & 1 & 208.4587 & 0.00000 \\
\hline LM Robusto (Lag) & 1 & 15.1310 & 0.00010 \\
\hline Multiplicador de Lagrange (Erro) & 1 & 200.9158 & 0.00000 \\
\hline LM Robusto (Erro) & 1 & 7.5881 & 0.00588 \\
\hline
\end{tabular}

Fonte: resultados da pesquisa. 
A estimação por MQO reportou coeficientes estatisticamente significativos (com 5\% de significância) para a eficiência técnica. O termo quadrático da variável demonstra que os municípios com escores muito altos de eficiência têm efeito negativo sobre o desmatamento. Já os municípios com escores pequenos proporcionam um aumento nos níveis de desmatamento, revelado pelo termo positivo do coeficiente da variável em nível.

O PIB municipal também apresenta sinal negativo, indicando que, quanto maior a produção do município, menor tende a ser seu potencial de desmatamento. Também se destaca como redutor do desmatamento o tamanho das propriedades, indicando que, quanto maiores os estabelecimentos rurais, menor será a área desmatada. O número de cabeças de gado bovino possui efeito positivo sobre o desmatamento, o que revela que a atividade pecuária na região tem contribuído para o desflorestamento.

Quanto aos diagnósticos de ajuste, o teste de Jarque-Bera de normalidade apresenta valor 2.75 ( $p$-valor 0.25 ) rejeita a hipótese nula, indicando uma distribuição normal dos erros, e o baixo $p$-valor do teste Breusch-Pagan demonstra a presença de heterocedasticidade. A condição de multicolinearidade menor do que 30 revela ausência de dependência entre regressores (Anselin \& Rey, 1981). Na sequência, os testes para dependência espacial indicam que a variável dependente é espacialmente autocorrelacionada. A significância estatística $(p$-valor $<0.01)$ dos testes $L M$-Lag e LM-Error indica que os modelos SAR e SEM possuem melhor ajuste do que o MQO. Além disso, valores superiores para log likelihood e inferiores para os critérios AIC e Schwarz, indicam que o modelo melhor ajustado é o de Erros Espaciais (SEM).

O modelo SEM promove um aumento na significância estatística dos estimadores de Escore, Escore ${ }^{2}$, Agrotóxicos, Eletricidade e Financiamento. Contudo, o PIB municipal deixa de ser relevante para explicar o desmatamento. Os coeficientes de dependência espacial ( $\rho$ para SAR e $\lambda$ para SEM) mostram-se estatisticamente significativos a $1 \%$. Além disso, enquanto os sinais dos estimadores permanecem inalterados em todos os modelos, suas magnitudes apresentam algumas alterações.

Dessa forma, os resultados das estimações indicam um efeito quadrático da eficiência técnica sobre o desmatamento, ou seja, o aumento dos escores de eficiência promovem um aumento nos níveis de desmatamento até um limite, a partir do qual, todo aumento na eficiência propicia uma redução do desmatamento. Esse padrão quadrático se mantém em todos os modelos, e vai de encontro aos 
resultados obtidos por Marchand (2012) ${ }^{4}$, que alcançou o extremo oposto. Em sua análise com dados de 1996, Marchand (2012) encontrou uma relação positiva de desmatamento e escores elevados de eficiência, enquanto que os agricultores com escores inferiores apresentaram relação negativa. Contudo, passadas duas décadas, verifica-se que a curva, que nos resultados de Marchand (2012) tinha forma de "U", passa a ter o formato inverso.

O turning-point calculado situa-se próximo ao nível de escore de eficiência técnica igual a 0.39 , indicando um ponto máximo no qual a eficiência técnica afetaria positivamente no desmatamento. Em nossa amostra, 609 municípios possuem eficiência abaixo desse limite. Portanto, a maior parte dos municípios (cerca de $72 \%$ do território estudado) situam-se na parte ascendente da curva, indicando que a eficiência técnica induz o aumento nos níveis de desmatamento.

Tomando por base os valores apresentados na Tabela 2, um aumento de $10 \%$ na eficiência média da Amazônia Legal (de 0.24 para 0.26) proporcionaria uma redução de 3\% na área desmatada, já que o efeito total da eficiência é negativo. Contudo, se houver melhora apenas na eficiência dos municípios com escore abaixo do turning-point (0.39), haverá aumento na área desmatada. Note que, se analisarmos a variação do desmatamento em relação à eficiência () temos que, para municípios com eficiência menor do que 0.39 , há efeito positivo sobre o desmatamento, enquanto que, para aqueles com escores superiores a 0.39 , o efeito é negativo.

Esses resultados levantam algumas questões importantes no que se refere às alternativas de soluções sustentáveis para a manutenção e/ou crescimento da produção agropecuária na região da Amazônia Legal. É evidente que o aumento da produção via uma persistente conversão de áreas florestais para agropecuárias já se considera insustentável, visto a grave contrapartida ambiental a que se está vulnerável.

O desafio das autoridades, portanto, é encontrar soluções regionalizadas para o problema da baixa eficiência técnica de maneira que não cause uma disparada nos níveis de desmatamento. É preciso que sejam implementadas políticas que permitam um grande salto de eficiência técnica agropecuária, uma vez que nas regiões em que os escores de eficiência são muito baixos, um aumento pequeno da eficiência levaria ao agravamento nos níveis de desmatamento.

4 Marchand (2012) avaliou o efeito dos escores de eficiência técnica agropecuária sobre a propensão a desmatar na ALB em 1996, utilizando o método de SFA para estimação dos coeficientes de eficiência, aplicando um modelo de uso da terra com estimação via Probit. 
Quanto à variável "área agropecuária", percebe-se que possui uma relação negativa e significativa com o desmatamento. $\mathrm{O}$ resultado sugere que municípios com estabelecimentos agropecuários menores tendem a desmatar proporcionalmente mais área do que aqueles em que há estabelecimentos maiores. Isso se dá pelo fato de que pequenos estabelecimentos, em geral, enfrentam maiores restrições técnicas e financeiras do que os grandes, fazendo com que se valham do fator abundante (terra), convertendo mais áreas de floresta em agropecuária.

\section{CONCLUSÕES}

A agropecuária apresenta elevada importância na economia brasileira, entretanto, tem sido alvo de muitos debates pelo ônus gerado ao meio ambiente, especialmente no que diz respeito à reversão de áreas florestais para a produção agrícola e pecuária. Diante disso, este trabalho analisou se, e de que forma, a eficiência agropecuária dos municípios da Amazônia Legal brasileira se relaciona com os níveis de desmatamento observados no ano de 2017.

Percebeu-se, a partir dos resultados obtidos, que a eficiência técnica contribui para o desmatamento de forma não-linear, de maneira que os municípios com pior desempenho em termos de eficiência agropecuária são os que mais contribuem para o aumento nos níveis de desmatamento. Por outro lado, os municípios com escores de eficiência mais elevados se correlacionam de forma negativa com o desmatamento, podendo contribuir para uma eventual redução nos prejuízos causados ao meio ambiente.

Além de demonstrar de que forma a eficiência agropecuária contribui para o desmatamento, também foi apresentada a distribuição espacial das variáveis e em quais regiões se deve dispensar maior atenção das autoridades e formuladores de políticas públicas visando a manutenção, ou mesmo um aumento, dos níveis de produção atentando-se para a atenuação dos danos ambientais causados pela expansão agropecuária sobre áreas florestais.

Políticas públicas voltadas para um aumento da eficiência nas regiões em que os escores são muito baixos, como é o caso da maioria dos municípios estudados $^{5}$, levaria a uma elevação nos níveis de desmatamento e devem, portanto, ser aplicadas em conjunto com medidas de proteção e fiscalização visando inibir

5 Ver Figura 2. 
o espalhamento das áreas agricultáveis para áreas de floresta. Já nas regiões em que se apresentam escores mais elevados, medidas que incentivem o aumento da eficiência produtiva podem atenuar os efeitos de desmatamento, já que para esse caso, a eficiência agropecuária se relaciona de forma inversa com o desmatamento.

Trabalhos futuros devem focar em métodos espaciais locais, como o de Regressão Ponderada Geograficamente, para averiguar a relação entre eficiência produtiva e desmatamento e determinar se os resultados servem para toda a amostra ou apenas para regiões específicas. Além disso, a incorporação de variáveis climáticas e de controle de efeitos fixos pode trazer melhor ajuste aos modelos.

\section{AGRADECIMENTO}

O presente trabalho foi realizado com apoio da Coordenação de Aperfeiçoamento de Pessoal de Nível Superior, Brasil (CAPES), Código de Financiamento 001.

\section{REFERÊNCIAS BIBLIOGRÁFICAS}

Almeida, C. A., Coutinho, A. C., Esquerdo, J. C. D. M., Adami, M., Venturieri, A., Diniz, C. G., Dessay, N., Durieu, L., \& Gomes, A. R. (2016). High spatial resolution land use and land cover mapping of the Brazilian Legal Amazon in 2008 using Landsat-5/TM and MODIS data. Acta Amazonica, 46(3), 291-302. Disponível em http://dx.doi.org/10.1590/1809-4392201505504

Almeida, E. (2012). Econometria espacial. São Paulo: Alínea.

Anselin, L. (1995). Local indicators of spatial association - LISA. Geographical analysis, 27(2), 93-115. Disponível em https://doi. org/10.1111/j.1538-4632.1995.tb00338.x

Anselin, L. (2005). Exploring spatial data with GeoDaTM: a workbook. [s.1.] CSISS. Disponível em http://www.csiss.org/clearinghouse/GeoDa/geodaworkbook.pdf

Anselin, L., \& Arribas-Bel, D. (2013). Spatial fixed effects and spatial dependence in a single cross-section. Papers in Regional Science, 92(1), 3-17. Disponível em https://doi.org/10.1111/j.1435-5957.2012.00480.x

Arraes, R. A., Mariano, F. Z., \& Simonassi, A. G. (2012). Causas do desmatamento no Brasil e seu ordenamento no contexto mundial. Revista de Economia e Sociologia Rural, 50(1), 119-140. Disponível em https://doi.org/10.1590/ S0103-20032012000100007 
Bacha, C. J. C. (2018). O papel da agropecuária no desenvolvimento econômico. Em: Economia e Política Agrícola no Brasil (pp. 241-266). São Paulo: Alínea.

Banker, R. D., Charnes, A., Cooper, W. W., \& Maindiratta, A. (1988). A comparison of DEA and translog estimates of production frontiers using simulated observations from a known technology. Em: Dogramaci A., \& Färe R. (eds.). Aplicaciones de la teoría de la producción moderna: eficiencia y productividad. Estudios de análisis de productividad (vol. 9). Dordrecht: Springer. Disponível em https://doi.org/10.1007/978-94-009-3253-1_2

Bhatt, M. S., \& Bhat, S. A. (2014). Technical efficiency and farm size productivity-micro level evidence from jammu \& kashmir. International Journal of Food and Agricultural Economics, 2(4), 27-49. Disponível em https://www. foodandagriculturejournal.com/2014\%20(vol2.no4).html

Charnes, A., Cooper, W. W., \& Rhodes, E. (1978). Measuring the efficiency of decision making units. European journal of operational research, 2(6), 429-444. Disponível em https://doi.org/10.1016/0377-2217(78)90138-8

Cliff, A. D., \& Ord, J. K. (1981). Spatial processes: models \& applications. S. 1.: Taylor \& Francis.

Coelli, T. J., Rao, D. S. P., \& Battese, G. E. (1998). An introduction to productivity and efficiency analysis. New York: Springer Science.

Coelli, T., Rahman, S., \& Thirtle, C. (2002). Technical, allocative, cost and scale efficiencies in Bangladesh rice cultivation: a non-parametric approach. Journal of Agricultural Economics, 53(3), 607-626. Disponível em https:// doi.org/10.1111/j.1477-9552.2002.tb00040.x

Debreu, G. (1951). The Coefficient of Resource Utilization. Utilization. Econometrica, 19(3), 273-292. doi:10.2307/1906814

Farrell, M. J. (1957). The measurement of productive efficiency. Journal of the Royal Statistical Society: Series A (General), 120(3), 253-281. Disponível em https://doi.org/10.2307/2343100

Food and Agriculture Organization (FAO). (2020) Production. Disponível em http://www.fao.org/faostat/en/\#data

Instituto Brasileiro de Geografia E Estatística (IBGE) (2020a). Amazônia Legal. Disponível em https://www.ibge.gov.br/geociencias/cartas-e-mapas/mapas-regionais/15819-amazonia-legal.html? $=\& \mathrm{t}=\mathrm{O}$-que-e

Instituto Brasileiro de Geografia E Estatística (IBGE) (2020b). Censo Agropecuário. Disponível em https://sidra.ibge.gov.br/pesquisa/censo-agropecuario/ censo-agropecuario-2017

Instituto Brasileiro de Geografia E Estatística (IBGE) (2020c). Pesquisa Pecuária Municipal e Produção Agrícola Municipal. Disponível em https://sidra.ibge. gov.br/ 
Koopmans, T. C. (1951). An analysis of production as an efficient combination of activities. Em: Activity analysis of production and allocation (pp. 625-628). London: Chapman and Hall.

Leibenstein, H. (1966). Allocative efficiency vs."X-efficiency". The American Economic Review, 56(30), 392-415. Disponível em https://www.jstor.org/ stable/1823775

Marchand, S. (2012). The relationship between technical efficiency in agriculture and deforestation in the Brazilian Amazon. Ecological Economics, 77, 166175. Disponível em https://doi.org/10.1016/j.ecolecon.2012.02.025

Oliveira, R. C. Almeida, E., da Silva Freguglia, R., \& Candéa Sá Barreto, R. (2011). Desmatamento e crescimento econômico no Brasil: uma análise da curva de Kuznets ambiental para a Amazônia legal. Revista de economia e sociologia rural, 49(3), 709-739. Disponível em https://doi.org/10.1590/S010320032011000300008

Silva, F., Perrin, R. K., \& Fulginiti, L. E. (2016). Tradeoffs between forests and farming in the Legal Amazon Region of Brazil. Em: 2016 Annual Meeting Southern Agricultural Economics Association (SAEA), San Antonio, Texas. Disponível em https://ageconsearch.umn.edu/record/230040/

Pacheco, P. (2009). Smallholder Livelihoods, Wealth and Deforestation in the Eastern Amazon. Ecologia humana, 37, 27-41. Disponível em https://link. springer.com/article/10.1007/s10745-009-9220-y

Stabile, M. C., Guimarães, A. L., Silva, D. S., Ribeiro, V., Macedo, M. N., Coe, M. T., Pinto, E., Moutinho, P., \& Alencar, A. (2020). Solving Brazil's land use puzzle: Increasing production and slowing Amazon deforestation. Land Use Policy, 91, 104-362. Disponível em https://doi.org/10.1016/j.landusepol.2019.104362

Tobler, W. R. (1979). Cellular geography. Em: Philosophy in geography (pp. 379386). Dordrecht: Springer.

Wadud, A., \& White, B. (2000). Farm household efficiency in Bangladesh: a comparison of stochastic frontier and DEA methods. Applied economics, 32(13), 1665-1673. Disponível em https://doi.org/10.1080/000368400421011

(C) 2021 por los autores; licencia no exclusiva otorgada a la revista Estudios económicos. Este artículo es de acceso abierto y distribuido bajo los términos y condiciones de una licencia Atribución-No Comercial 4.0 Internacional (CC BY-NC 4.0) de Creative Commons. Para ver una copia de esta licencia, visite http://creativecommons.org/licenses/by-nc/4.0 